\title{
Results and long-term patient satisfaction after gluteal augmentation with platelet-rich plasma-enriched autologous fat
}

\author{
Joep C. N. Willemsen • Nicole Lindenblatt • \\ Hieronymus P. J. D. Stevens
}

Received: 2 May 2013 / Accepted: 7 August 2013 / Published online: 1 September 2013

(C) Springer-Verlag Berlin Heidelberg 2013

\begin{abstract}
Background Buttock augmentation is gaining increasing popularity in aesthetic surgery. The relatively high incidence of complications after silicone implant placement lead to the increased use of lipofilling techniques, yielding variable results with respect to graft take rate and long-term stability. Platelet-rich plasma (PRP) has been shown to have beneficial effects on wound healing and angiogenesis in the past. Therefore, we aimed at investigating the long-term results and patient satisfaction after PRP-enriched lipofilling for buttock augmentation.

Methods Twenty-four bilateral gluteal augmentations with PRP-enriched autologous fat were performed. Additionally, contour shaping was achieved by liposuction of the adjacent zones. Post-operative results and complications were recorded, and satisfaction with buttock shape was estimated by a patient questionnaire.

Results Mean follow-up time was 44 months, and mean amount of transferred fat was $481 \mathrm{cc}$ for both sides. No seroma or hematoma formation, infection or liponecrosis were reported during the post-operative follow-up. Subjective patient satisfaction in general increased from preoperatively to 3 months postoperatively and declined only slightly in the long-term course. Satisfaction levels in general were specific for each patient. Patient recovery was quick, and the majority of patients returned to work within 10 days after surgery.
\end{abstract}

Joep C.N. Willemsen and Nicole Lindenblatt contributed equally to the manuscript

J. C. N. Willemsen • H. P. J. D. Stevens

Bergman Clinics The Hague, Binckhorstlaan 149, 2516 BA The

Hague, The Netherlands

N. Lindenblatt $(\bowtie)$

Division of Plastic and Hand Surgery, Department of Surgery,

University Hospital Zurich, Raemistrasse 100, 8091 Zürich,

Switzerland

e-mail: niclindenblatt@hotmail.com
Conclusions PRP-enhanced lipofilling of the buttocks proved to be a safe procedure including a low complication rate and consistent results. However, subjective patient expectations have to be taken into account when choosing the indication. Further large volume studies are needed to elucidate the potential and benefit of PRP in this context.

Level of Evidence: Level IV, therapeutic study.

Keywords Lipofilling $\cdot$ PRP $\cdot$ Buttocks $\cdot$ Gluteal augmentation $\cdot$ Fat

\section{Introduction}

Over the past decades, the demand for a better definition of the body and the buttock area has been increasing [1]. Augmenting the buttocks by placement of implants represents a commonly used technique. The relatively high incidence of implant-related complications, e.g. infection, malpositioning, nerve compression, wound healing problems or unnatural aesthetic appearance, lead to the quest for alternative techniques [2]. In the 1980s, liposuction was introduced, making fat asservation and reinjection to correct contour irregularities possible, and subsequently, buttock augmentation by lipofilling was performed $[3,4]$. The effect on lower body contouring was even improved by the combination of gluteal lipofilling and liposuction of the adjacent areas of lower back and thighs [5].

One drawback of this procedure is that it may have to be repeated several times to achieve the desired result due to fat resorption, leading to increased costs and risks for the patients. On the other hand, injecting too large volumes of fat may result in an increase of complications, such as seroma formation, liponecrosis, infection and fat embolism syndrome [2, 4]. Therefore, efforts to improve fat graft take have to be made. One approach is the addition of previously prepared adiposederived stem cells (ADSCs) to the fat graft, which is referred to 
as cell-assisted lipotransfer (CAL). ADSCs are isolated with a specific machine converting the aspirated fat into ADSC-rich fat. First studies were able to show that CAL is safe for soft tissue augmentation and leads to stable volumes over 2 months $[6,7]$. However, the application of this method at the moment still necessitates the use of specialized expensive equipment, making it less attractive. Also, the safety of the injection of potentially mutagenic cells has not finally been proven [8].

Another promising strategy may be the addition of plateletrich plasma (PRP) to the fat graft. PRP represents a plasma fraction exhibiting a platelet concentration above baseline values [9]. After activation, platelets synthetize and secrete a number of cytokines and growth factors, the most important ones being platelet-derived growth factor (PDGF), transforming growth factor beta-1 (TGF- $\beta 1)$, vascular endothelial growth factor (VEGF) and epidermal growth factor (EGF) $[10,11]$.

In the last decade, several smaller studies were performed, demonstrating the positive effect of PRP on wound healing, the formation of granulation tissue and neo-vascularization [10-15]. In a recent systematic review, a potentially beneficial effect of PRP for several indications, including wound healing, survival rate of fat grafts and an enhancement of bone graft regeneration, has been proposed [11]. Considering the potential pro-angiogenic effects of PRP, the addition of PRP in buttock augmentation appears to be a promising alternative to improve fat graft take and subsequently long-term results and graft stability.

Based on these facts, it was the aim of this study to evaluate results and long-term patient satisfaction of PRP-enhanced gluteal lipofilling of the buttocks in our patient collective.

\section{Material and methods}

\section{Patient characteristics}

Twenty-four gluteal bilateral augmentations were performed in 21 healthy female patients by the senior author from 2004 to 2012. In all cases, the indication for the procedure was aesthetic. Lack of volume and projection was the main concern of the patients and reason for the consultation. The group did not differ significantly in age, smoking behaviour or body mass index, and no patient underwent any other operation in the buttock area. Mean age of the patients at the time of operation was 42.8 years (range, 21-69 years). In average, patients presented with a normal body mass index of $24.7 \pm 0.9 \mathrm{~kg} / \mathrm{m}^{2}$.

\section{Study design}

Informed consent of all patients was given. Permission to conduct this study was granted, and the study was approved by the Institutional Review Board of the Medical Clinic.
Standardized pre- and post-operative photos were taken of all patients. In February 2013, all patients were contacted again and asked to fill out a patient questionnaire comprising ten questions concerning satisfaction with the operative results and post-operative experience of adverse events. Additionally, all patient charts were retrospectively screened for the occurrence of complications of any kind. Patient satisfaction was rated on a scale from 1 to $10(1=$ very dissatisfied, $5=$ neutral, $10=$ extremely satisfied) before the operation, 3 months after the operation and at present time. Additionally, patients were questioned regarding weight change, recurrent or persisting swelling of the buttocks, changes in skin quality and appearance, tissue hardening, buttock shape and side differences. Patients were asked whether they would consider another lipofilling session to even improve outcome or recommend the procedure to other people.

Follow-up lipofilling with PRP buttocks

1. How satisfied were you with the shape of your buttocks before the operation?

Rate on a scale from 1 to $10(1=$ very dissatisfied, $5=$ neutral, $10=$ extremely satisfied)

2. How satisfied were you with the shape of your buttocks 3 months after the operation?

Rate on a scale from 1 to $10(1=$ very dissatisfied, $5=$ neutral, $10=$ extremely satisfied)

3. How satisfied are you with the shape of your buttocks today?

Rate on a scale from 1 to $10(1=$ very dissatisfied, $5=$ neutral, $10=$ extremely satisfied)

4. Did you lose or gain weight after the operation permanently? If yes, how much?

5. Did you experience continuous or recurrent swelling of the buttock region 3 months after the operation?

6. Did you experience changes in the skin appearance and quality 3 months after the operation? If yes, which?

7. Did you notice hardening of the fat tissue of the buttocks 3 months after surgery?

8. Did you notice a difference of shape between both sides of the buttocks 3 months postoperatively?

9. Would you consider another buttock lipofilling operation to improve results?

10. Would you recommend buttock augmentation with autologous fat to other people?

Fat harvest and lipofilling

For the harvesting of the fat, the standard Coleman procedure was used [16]. Briefly, stab incisions of $2 \mathrm{~mm}$ were made to infiltrate after which harvesting of fat was performed with the 
original Coleman harvesting cannula. The areas where the fat was harvested include flanks, abdomen, subgluteal and trochanteric areas, knees, legs and lateral and inner thighs. Approximately two to three times more fat was harvested than the estimated amount required for injection. Fat was centrifuged for $3 \mathrm{~min}$ at the maximum speed of 3,000 rpm (IEC MediSpin Centrifuge), after which the oil (top layer) and serum/infiltrate layers (bottom layer) were discarded, preserving the preadipocyte-rich pellet [17]. After centrifugation, the middle layer consisting of firm adipose tissue was used. Subsequently, extensive deep and superficial liposuction of the lower back was performed, using a 3-mm accelerator type cannula with three holes on one side. This allowed for volume reduction and very superficial liposuction, ensuring contraction and lifting of the buttock.

Injection of harvested fat as described above was performed in a preoperatively predefined area in each patient in a banana-shaped pattern into the buttocks. Fat was introduced mainly in the subcutaneous area; a small portion of the fat was injected into the gluteus muscles. The PRP was added to the last $60 \mathrm{cc}$ of fat to be injected and then dispersed evenly throughout the entire target zone. By this, an evenly distributed effect of PRP was anticipated. Injection into the buttock target zone was performed by dispersing the enriched fat equally, spreading the PRP-containing fraction in the more superficial layers underneath the buttock skin.

\section{Preparation of PRP}

Blood was drawn from the patient preoperatively, and $2 \times$ $54 \mathrm{cc}$ whole blood was introduced in the Biomet GPS-III device after adding $6 \mathrm{cc}$ of citrate to prevent clothing (Biomet Biologics Inc., Warsaw, IN., USA). Fifteen minutes of centrifugation at 3,500 rpm allowed for gravitational separation of the whole blood into its three fractions: erythrocytes, platelet poor plasma (PPP) and PRP. A total of $12 \mathrm{cc}$ of PRP was yielded in this fashion. Activation of PRP resulting in degranulation and subsequently release of the growth factors can be achieved either by adding thrombin or calcium. In this series, autologous thrombin was used, obtained from a whole blood sample of the patient. A PRP of $6 \mathrm{cc}$ was added to the last $60 \mathrm{cc}$ of fat to be injected, and the fat was evenly dispersed within the infiltrated area.

\section{Post-operative treatment}

All patients wore a specifically fitted compression garment for the lower back, buttocks and thighs over a period of 68 weeks. No specific post-operative limitations were felt necessary nor advised. No lipofilling was performed on top of the Tuber ischiadicum, so patients were allowed to sit normally directly after surgery.
Statistical analysis

After proving the assumption of normality and equal variance across groups, differences between groups were assessed using one-way ANOVA followed by the appropriate posthoc comparison test. Mann-Whitney $U$ tests were applied for non-parametric distributions. Overall statistical significance was set at $p<0.05$. Values are given as mean and SEM. Statistics and graphics were performed using the software packages SigmaStat and SigmaPlot (Jandel Corporation, San Rafael, CA, USA).

\section{Results}

The mean follow-up time in this study was 44 months (range, 3-106 months). Mean injection volumes for both sides were $481 \mathrm{cc}$ (range, 28-720 cc). In the post-operative course, no events of infection, cellulitis or seroma formation were reported. Next to this, no cases of liponecrosis were found. Deep vein thrombosis or pulmonary embolism did not occur. One patient developed a thrombophlebitis of the arm in which the intravenous needle was placed, which was managed conservatively.

\section{Post-operative questionnaire}

Nine out of 21 patients ( $43 \%$ ) completed the post-operative questionnaire. In this patient group, the follow-up amounted to 37 months at the time of the survey (range, 3-60 months). Preoperatively, patients judged the appearance of their buttocks in average with a value of $3.6 \pm 1.0$ on a scale from 1 to 10 (Fig. 1a). Three months postoperatively, the satisfaction increased to a mean value of $6.1 \pm 1.0$ and dropped again slightly to $5.3 \pm 1.3$ at present $(p=$ n.sig.). The average subjective patient rating thus tended to increase from less satisfied to a value above midline indicating increased satisfaction. Two patients still rated the result at present as "very dissatisfied". However, these patients also presented with a low satisfaction value preoperatively (Fig.1b). Two patients experienced a marked increase in satisfaction with the result after 3 months, which was not maintained on long-term, indicating a loss of volume over time. More detailed analysis revealed that patients in fact experienced very individual courses of satisfaction with the post-operative results (Fig. 1b). While some patients stated to be very dissatisfied before the operation without change after the gluteal augmentation, some patients experienced a strong increase in satisfaction with the shape of their buttocks. In general, it is striking that the post-operative estimation level generally is in line with the preoperative judgement, i.e. patients with a low satisfaction level preoperatively tended to stay on this level, while patients that were already quite satisfied 
A

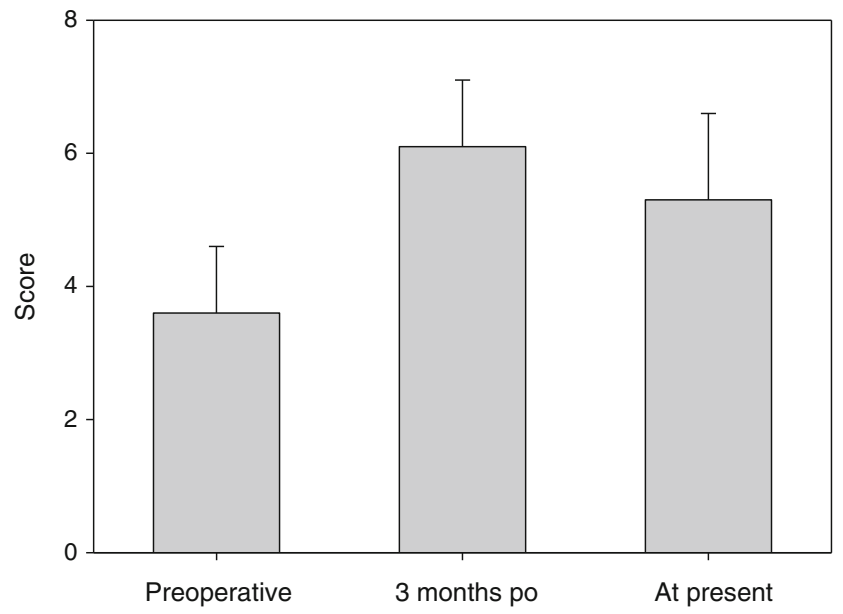

Fig. 1 a Satisfaction levels preoperatively, 3 months postoperatively and at present with appearance of the buttocks on a scale from 1 to $10(1=$ very

with the appearance of their buttocks rated as attractive also after the operation (Fig. 1b). Two out of nine patients gained weight after the operation. However, weight gain did not correlate with post-operative satisfaction with the result. One patient out of nine reported recurrent post-operative swelling at 3 months after the operation; the other eight patients did not have these complaints. No patients observed a change in skin quality, neither for better nor for worse, or dimpling of the skin. Two out of nine patients reported several hardened areas over the buttocks at 3 months postoperatively. Slight contour differences between both buttocks were noted by four out of nine patients at 3 months postoperatively. Of note, all patients would undergo the same operation again. Six out of nine patients would also recommend the operation to others or were neutral concerning this. Three patients underwent a second lipofilling procedure of the buttock region to increase volume. Patient recovery was quick; the majority of patients in this series returned to work within 10 days after surgery.
B

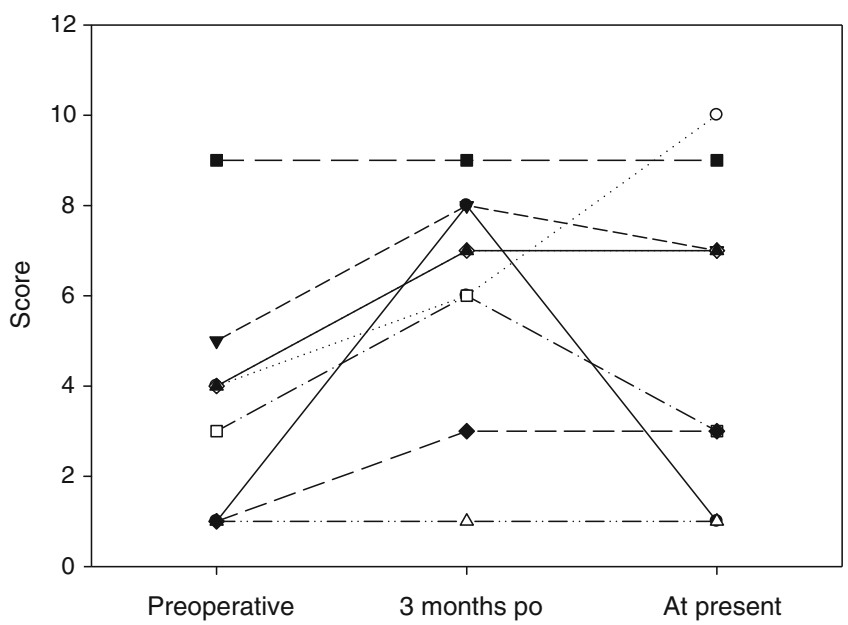

dissatisfied, $5=$ neutral, $10=$ extremely satisfied). b Evolution of

Case report 1

A 36-year-old woman presented to our clinic because of dissatisfaction with the contour of the buttock, flanks, abdomen and upper legs. She had finally lost $7 \mathrm{~kg}$ being stable for more than 4 months. Despite an average of 2-3 h of physical workout weekly, she could not achieve a better shape of her body contour. On physical examination, we saw a healthy young woman with a height of $1.75 \mathrm{~m}$ and a weight of $68 \mathrm{~kg}$. There was an obvious hypovolemia of the buttocks that was accompanied by local fat deposition on the flanks and legs (Fig. 2a). We proposed lipofilling with PRP of the buttocks combined with an extensive liposuction procedure of the back, flanks, abdomen and upper legs. A total of $210 \mathrm{cc}$ of fat cells (after centrifuging) were injected per buttock, and a total of 2,800 cc of liposuction was performed on legs, abdomen and back. Wound healing was uncomplicated. The contour of the entire body improved significantly, leading to a high patient satisfaction (Fig. 2b).
Fig. 2 a Preoperative and $\mathbf{b}$ 13 months postoperative results in pa and lateral view of a patient after lipofilling with $420 \mathrm{cc}$ and PRP of both buttocks and contouring liposuction of legs, abdomen and back
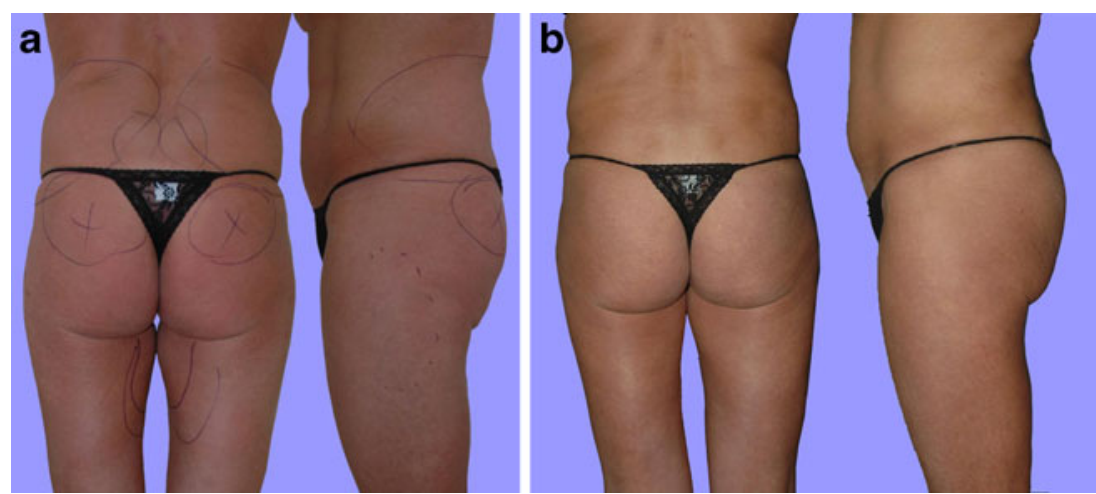
Fig. 3 a Preoperative and b 12 months postoperative results in pa and lateral view of a patient after lipofilling with $700 \mathrm{cc}$ and PRP of both buttocks and contouring liposuction of lower back, abdomen, lateral, anterior and medial part of the leg
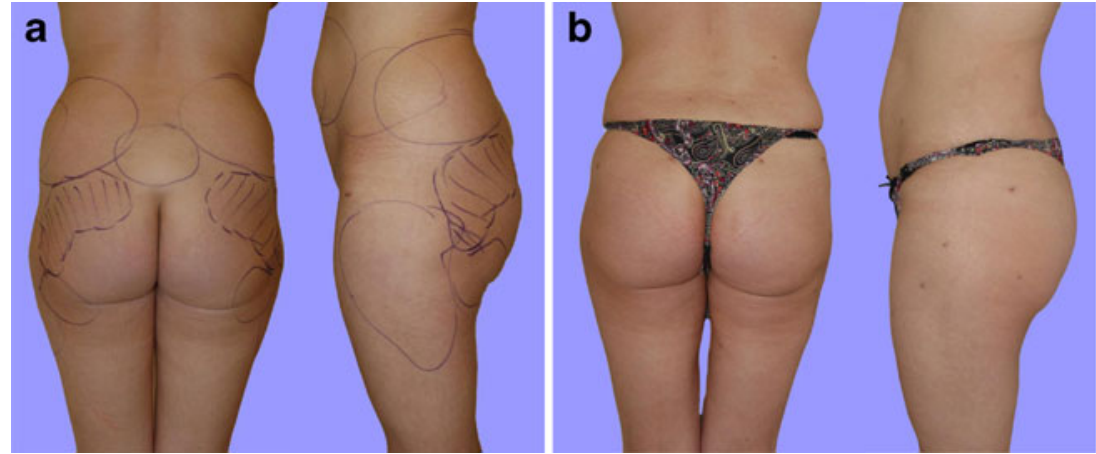

\section{Case report 2}

A 32-year-old woman presented with a rather square-shaped figure that she considered displeasing. She was dissatisfied with the fullness of lower back, abdomen and upper legs (Fig. 3a). Previous liposuction of the abdomen elsewhere did not yield a satisfactory result. Liposuction specifically targeting to the lower back, abdomen, lateral, anterior and medial part of the legs was performed under general anaesthesia, removing 2,400 cc of fat. One thousand one hundred cubic centimetres of fat were harvested with refined cannulas by syringe liposuction. The former was centrifuged, generating $350 \mathrm{cc}$ of fat for injection per buttock, evenly distributed as fat droplets in both superficial as well as deeper layers, from subcutis into the gluteal muscle. Six cubic centimetres of autologous PRP was added per buttock. The patient was very satisfied with the result of this procedure. Results proved to be stable over 1-year follow-up (Fig. 3b).

\section{Discussion}

Over the past decade, autologous fat grafting has gained wide popularity due to its good availability, autologous nature, volume and beneficial regenerative effects [18]. However, survival of the transplanted fat cells still remains unpredictable, fuelling the continuous search for technical refinement and improvement of fat graft take. Popularized by Coleman lipofilling also was applied in body contouring [16]. Large series are available in literature, describing several techniques with varying volumes. One of the first large series $(n=879)$ was described by de Pedroza using volumes up to $350 \mathrm{cc}$ of autologous fat for buttock augmentation [19]. In the whole series, he reported only one complication (one infection) and described no loss of volume or graft over time. After increase of the grafted volume, complications like liponecrosis, infection and lack off buttock projection increased significantly [20]. Several cases of the potentially life-threatening fat embolism syndrome were reported $[1,20]$. In a study of Nicareta et al., a high patient satisfaction is reported within the first months after surgery and after several years [4]. In this study, the importance of extensive and objective preoperative consultation as an important factor in achieving a more satisfied patient was stressed. The study of Cárdenas-Camarena et al. is the only available study that reports on patient satisfaction among different techniques (implants and lipofilling) [20]. This study showed no significant difference between the percentage of satisfied patients when comparing augmentation with implants versus augmentation with lipofilling.

To allow for even larger volumes to be injected in gluteal augmentation, one promising strategy may be the addition of PRP to the fat graft. PRP is a concentration of autologous human platelets in a small volume of plasma, containing seven main growth factors degranulated by platelets after activation (PDGF- $\alpha \alpha$, PDGF- $\alpha \beta$, PDGF- $\beta \beta$, TGF- $\beta 1$ TGF- $\beta 2$, VEGF, EGF) $[9,10]$. These factors are capable of enhancing cell proliferation, differentiation and neoangiogenesis [21, 22]. Several animal studies reported improved graft take after addition of PRP with a higher number of viable adipocytes and infiltrating blood vessel found histologically [14, 23]. PRP will also release a wide range of growth factors that play a key part in tissue regeneration, which could boost local tissue repair and shortening of recovery time [10]. In particular, PDGF-AB and TGF-b1 significantly promote the proliferation of ADSCs and dermal fibroblasts [24].

However, the beneficial properties of PRP in the context of lipofilling with the intent to achieve rapid vascularization and stable long-term results are far from established [11, 25]. Available studies reported enhanced wound healing after PRP-enriched fat grafting [15, 26]. Cervelli et al. presented results from patients treated for facial soft tissue aging. They showed that the use of PRP during fat grafting improves adipose tissue maintenance and survival when compared with fat grafting alone [26]. On the other hand, no enhancement of fat graft take in the breast after enrichment with PRP when compared with fat grafting alone was observed [22]. The authors even observed a higher rate of liponecrosis in patients treated with PRP, even if this was not statistically significant. There are no studies evaluating the effect of PRP-enriched lipofilling of the buttocks at the moment.

We herein report a case series of 24 bilateral PRP-enriched lipofillings of the buttocks. The general complication rate with respect to acute adverse events, such as infection, fat or 
pulmonary embolism, was very low, and no major complications were identified. No obvious case was noted for liponecrosis, a complication that has been reported recently in PRP-enriched lipofilling of the breast [22]. The transferred volumes used in this study were not as high as some reports in literature since the demand for such large amount of volumes is not common in our European country. Moreover, we did not aim at overcompensating for fat graft loss because of the use of PRP. Patient recovery and healing were fast. In order to learn more about the long-term results and patient satisfaction, we performed a survey among the patients. This survey clearly showed patient expectations and satisfaction very distinctly in buttock augmentation. While objective results were good and the general complication rate was very low, patients themselves exhibited individual grades of satisfaction with the result. The subjective happiness with the results tended to be only slightly higher at 3 months after the operation than on long-term. The high success rate and the absence of complications as fat necrosis may be due to the addition of PRP in combination with a more appropriate quantity of injected fat cells in relation to the amount of vascularization of the target zone. PRPenriched fat grafting may therefore represent a feasible alternative to immediate cell-assisted lipotransfer [27].

\section{Conclusion}

In the presented study, we report that PRP-enhanced lipofilling of the buttocks proves to be a safe procedure, entailing a low complication rate. However, patient expectations have to be taken into account and most probably, several sessions will be necessary to achieve the desired volume in some patients. Larger prospective randomized studies evaluating the effect of PRP on gluteal liposculpture, in particular in comparison to lipofilling alone, should be performed in the future to assess the potential beneficial effect on volume persistence.

\section{Conflict of interest None.}

\section{References}

1. Cardenas-Camarena LR, Arenas-Quintana R, Robles-Cervantes RA (2011) Buttocks fat grafting: 14 years of evolution and experience. Plast Reconstr Surg 128:545-555

2. Bruner TW, Roberts TL 3rd, Nguyen K (2006) Complications of buttocks augmentation: diagnosis, management, and prevention. Clin Plast Surg 33:449-466

3. llouz YG (1986) The fat cell "graft": a new technique to fill depressions. Plast Reconstr Surg 78:122-123

4. Nicareta B, Pereira LH, Sterodimas A, Illouz YG (2011) Autologous gluteal lipograft. Aesthetic Plast Surg 35:216-224

5. Gasparotti M (1992) Superficial liposuction: a new application of the technique for aged and flaccid skin. Aesthetic Plast Surg 16:141-153
6. Sterodimas A, De Faria J, Nicaretta B, Papadopoulos O, Papalambros E, Illouz YG (2010) Cell-assisted lipotransfer. Aesthet Surg J 30:78-82

7. Yoshimura K, Sato K, Aoi N, Kurita M, Hirohi T, Harii K (2008) Cellassisted lipotransfer for cosmetic breast augmentation: supportive use of adipose-derived stem/stromal cells. Aesthetic Plast Surg 32:4-55

8. Choi J, Minn KW, Chang H (2012) The efficacy and safety of platelet-rich plasma and adipose-derived stem cells: an update. Arch Plast Surg 39:585-592

9. Marx R, Carlson E, Eichstaedt R, Schimmele S, Strauss J, Georgeff K (1998) Platelet-rich plasma: growth factor enhancement for bone grafts. Oral Surg Oral Med Oral Pathol Oral Radiol Endod 85:638-646

10. Eppley B, Pietrzak W, Blanton M (2006) Platelet-rich plasma: a review of biology and applications in plastic surgery. Plast Reconstr Surg 118:147-159

11. Sommeling CE, Heyneman A, Hoeksema H, Verbelen J, Stillaert FB, Monstrey S (2013) The use of platelet-rich plasma in plastic surgery: a systematic review. J Plast Reconstr Aesthet Surg 66:301-311

12. Lacci KM, Dardik A (2010) Platelet-rich plasma: support for its use in wound healing. Yale J Biol Med 83:1-9

13. Pires Fraga MF, Nishio RT, Ishikawa RS, Perin LF, Helene A Jr, Malheiros CA (2010) Increased survival of free fat grafts with plateletrich plasma in rabbits. J Plast Reconstr Aesthet Surg 63:e818-e822

14. Nakamura S, Ishihara M, Takikawa M, Murakami K, Kishimoto S, Nakamura S, Yanagibayashi S, Kubo S, Yamamoto N, Kiyosawa T (2010) Platelet-rich plasma (PRP) promotes survival of fat-grafts in rats. Ann Plast Surg 65:101-106

15. Cervelli V, Gentile P, Scioli MG, Grimaldi M, Casciani CU, Spagnoli LG, Orlandi A (2009) Application of platelet-rich plasma in plastic surgery: clinical and in vitro evaluation. Tissue Eng Part C Methods 15:625-634

16. Coleman SR (2006) Structural fat grafting: more than a permanent filler. Plast Reconstr Surg 118(3 Suppl):108S-120S

17. Piasecki JH, Gutowski KA, Moreno KM, Lahvis GL (2008) Purified viable fat suspended in matrigel improves volume longevity. Aesthet Surg J 28:24-32

18. Rigotti G, Marchi A, Galiè M, Baroni G, Benati D, Krampera M, Pasini A, Sbarbati A (2007) Clinical treatment of radiotherapy tissue damage by lipoaspirate transplant: a healing process mediated by adipose-derived adult stem cells. Plast Reconstr Surg 119:1409-1422

19. de Pedroza LV (2000) Fat transplantation to the buttocks and legs for aesthetic enhancement or correction of deformities: long-term results of large volumes of fat transplant. Dermatol Surg 26:1145-1149

20. Cardenas-Camarena L, Silva-Gavarrete JF, Arenas-Quintana R (2011) Gluteal contour improvement: different surgical alternatives. Aesthetic Plast Surg 35:1117-1125

21. Hausman GJ, Richardson RL (2004) Adipose tissue angiogenesis. J Anim Sci 82:925-934

22. Salgarello M, Visconti G, Rusciani A (2011) Breast fat grafting with platelet-rich plasma: a comparative clinical study and current state of the art. Plast Reconstr Surg 127:2176-2185

23. Oh DS, Cheon YW, Jeon YR, Lew DH (2011) Activated platelet-rich plasma improves fat graft survival in nude mice: a pilot study. Dermatol Surg 37:619-625

24. Kakudo N, Minakata T, Mitsui T, Kushida S, Notodihardjo FZ, Kusumoto K (2008) Proliferation-promoting effect of platelet-rich plasma on human adipose-derived stem cells and human dermal fibroblasts. Plast Reconstr Surg 122:1352-1360

25. Freshwater MF (2013) The use of platelet-rich plasma in plastic surgery remains unproven. J Plast Reconstr Aesthet Surg 66:311-312

26. Cervelli V, Gentile P, De Angelis B, Calabrese C, Di Stefani A, Scioli MG, Curcio BC, Felici M, Orlandi A (2011) Application of enhanced stromal vascular fraction and fat grafting mixed with PRP in posttraumatic lower extremity ulcers. Stem Cell Res 6:103-111

27. Condé-Green A, Lamblet H (2012) Immediate cell-supplemented lipotransfer (ICSL). Eur J Plast Surg 35:373-378 